\title{
Erratum to: Congenital Esophageal Stenosis Associated with Esophageal Atresia
}

\author{
Ashraf H.M. Ibrahim and Talal A. Al Malki
}

\section{Erratum to:}

Chapter 9 in: H. Till et al. (eds.), Esophageal and Gastric Disorders in Infancy and Childhood, DOI 10.1007/978-3-642-11202-7_9

The affiliation of Dr. Ibrahim was incorrect.

The correct affiliation of Ashraf H.M. Ibrahim is given below:

A.H.M. Ibrahim

King Faisal Military City

P. O. Box: 5062

Khamis Mushait

Saudi Arabia

The updated original online version for this chapter can be found at DOI 10.1007/978-3-642-11202-7_9

A.H.M. Ibrahim, FRCSI, MD $(\bowtie)$

King Faisal Military City, P. O. Box: 5062,

Khamis Mushait, Saudi Arabia

e-mail: ash_ib@hotmail.com

T.A. Al Malki, FRCSC

Faculty of Medicine, Al-Taif University,

Taif, Saudi Arabia 